\title{
Further Exploration of Necrotic Control of Evolved Art
}

\author{
Daniel Ashlock and Julie Greensmith
}

\begin{abstract}
This study is the second in investigating the use of necrosis based filtration as a method of steering evolutionary algorithms to create evolved art. We use a technique inspired by the danger theory of immune system activation - a method employed in Artificial Immune Systems. An earlier study tested two types of filters used to augment the evolution of apoptotic cellular automata, shape and state diversity, applying one as a soft filter and the other as a crisp filter. This study tests both shape and state diversity filters in both soft and crisp modes and also mixes the filters, applying two filters in a single evolutionary algorithm. Soft and crisp version of the same filter are found to have substantially different behavior. The stacking of filters is found to work simply and transparently, effectively excluding undesirable parts of the fitness landscape.
\end{abstract}

\section{INTRODUCTION}

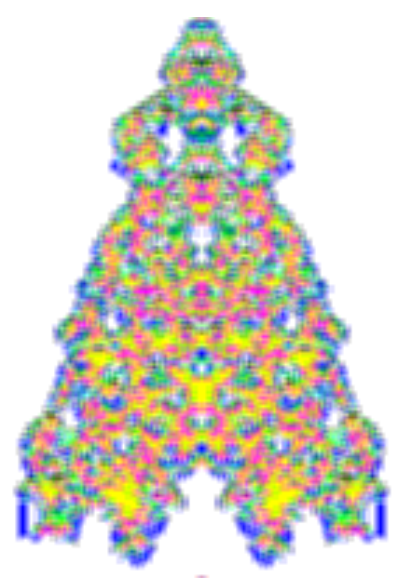

Fig. 1. An example of an evolved apoptotic cellular automata steered toward diverse color use by necrotic filters.

This study continues work from [5] on using a techniques from Artificial Immune Systems[16] to modify the behavior of an evolutionary algorithm, restricting the type of optima that the algorithm can locate. The target of the study are apoptotic cellular automata [7], a type of self-delimiting image that is an example of evolved art [6]. An example of this type of cellular automata appears in Figure 1. In the earlier study, two types of restrictions were applied to the evolution of apoptotic cellular automata, a soft restriction on the shape of the rendered picture, and a crisp restriction of the entropy of the distribution of colors that appeared in the images. The restrictions were applied using what is termed a 'necrosis technique' derived from the concept of

Daniel Ashlock is with the Department of Mathematics and Statistics at the University of Guelph, in Guelph, Canada email: dashlock@uoguelph.ca

Julie Greensmith is with the School of Computer Science at the University of Nottingham, UK, email: Julie.Greensmith@nottingham.ac.uk

The authors thank the Universities of Guelph and Nottingham for their support of this work the danger theory employed by Artificial Immune Systems [13]. The choice of soft and crisp applications of the necrosis filter was initially arbitrary in [5] and it was noted as an extension to the research that multiple necrosis filters could be applied simultaneously. This study examines both crisp and soft versions of necrotic filtering for shape and entropy restrictions and experiments with applying multiple necrosis filters in a single collection of evolutionary runs.

With the advent of Artificial Immune Systems as computational intelligence techniques, a number of methods inspired by natural immune systems have been added to the array of available methods for nature inspired computation. This study and the one it builds on apply the immune-system inspired concepts of the danger theory[19]. This is the body's ability to discriminate between two types of cell death: necrosis and apoptosis. Apoptosis is planned and controlled cell death, used to regulate tissue growth. Necrosis is the violent disruption of a cell caused by some form of stress, leading to its recognition by the immune system as being associated with foreign material as part of the detection of non-self entities. In this process the infected cell itself gives off biochemical signals representing danger or damage to tissue cells. In a departure from the conventional use of danger signals in dendritic cell based Artificial Immune Systems[15], in this immune system-inspired metaphor population members emit danger signals upon reaching a set criteria for necrosis. In this research, necrosis is used to create a novel computational intelligence technique by adding a necrotic step as a filter to an existing evolutionary algorithm. Necrosis zeros out the fitness of population members with undesirable properties. This is presented as an alternative to trying to write complex fitness function that bake in constraints into a single feature of the algorithm, as was done in [8].

Cellular automata are a type of discrete model of computation. A cellular automata has three parts,

1) A collection of cells divided into neighborhoods of each cell. In this study the cells form a linear array of cells. Neighborhoods consist of a cell and its four nearest neighbors.

2) A set of states that can be taken on by cells. In this study we use the numbers 0-7 as states.

3) A rule that maps the set of possible cell states of a neighborhood to a new state for the neighborhood. This rule is described subsequently and is the target of evolution.

Cellular automata (CA) can be described as discrete dynamical systems that exhibit self-organizing behaviour. The cells states evolve according to local transition rules. The up- 
dating according to the transition rules may be synchronous or asynchronous with apoptotic cellular automata using synchronous updating. Cellular automata can be used as models of complex natural systems that contain large numbers of identical components experiencing local interactions [26], [23].

Cellular automata have been applied to the study of a diverse range of topics, such as structure formation[11], heat conduction[12], language recognition[21], traffic dynamics[18] and cryptography[2], to name a few. CA have also been used for more aesthetic purposes, such as image and sound generation. Serquera and Miranda of the Interdisciplinary Centre for Computer Music Research, UK, have published on the use of CA for sound synthesis [25], [1].

CA have been applied to the visual arts, being used to produce artistic images[9], [20], [8], [14], in the form of time histories of one-dimensional cellular automata with states shown as colors. The automata in this study are used in the same fashion. Two dimensional automata have been used to produce level maps for games [17], [3], [4]. This work demonstrated that CA can generate not just a single map but families of maps with similar properties. Ashlock and Tsang[9] produced evolved art using 1-dimensional CA rules. CA rules were evolved using a string representation. The CA either underwent slow persistent growth, or planned senescence. The resulting fitness landscapes were rugged with many local optima. This led to the production of aesthetically pleasing images. Another common target for evolved art is fractals [6] and one of the reviewers thought our CAs were fractals: they are not.

In this study we return to earlier work which demonstrated that the fitness landscape of even simple encoding of CA used in this study have a complex fitness landscape [7]. In [8] the algorithm for finding CA rules was modified to restrict the shape of the rendered images. This study extends the number of techniques that employ necrosis to eliminate undesirable images. Necrosis filters are applied to the rendered images derived from the $\mathrm{CA}$ rules, meaning that the selection criteria applied by the necrosis filters can be specified in a manner directly related to the appearance desired by the user. The selection criteria used are a desired shape for the image and bounds on the entropy of state usage, both upper and lower.

The rest of this study is structured as follows. In Section III we give the design of experiments, including the representation, fitness functions, and analysis tools. In Section IV the results are presented and discussed. Section V we draw conclusions and discuss potential next steps.

\section{Apoptosis, Necrosis And THE DAnger Theory}

Artificial Immune Systems (AIS) emerged in the 1990s as a novel computational technique[16], centered around the elegant idea of creating a computer immune system to detect computer viruses. As these techniques have matured, different parts of the behaviour of the human immune system have been incorporated. AIS are a diverse collection of algorithms, all based on metaphors of different aspects of the function and behaviours of the immune system. Initial approaches in AIS focused on the principles of self-nonself, where the adaptive immune system is used as inspiration in the development of classical-style supervised learning paradigms.

As the field evolved, contemporary theories in immunology featured more prominently in AIS. One such immunological phenomena, the danger theory, gained traction in immunology as a credible mechanism by which the human immune system decides if and when to mount an immune response against a pathogen. While still controversial in immunology, from 2002 the danger theory started to feature in AIS research. In a departure from the standard self-nonself based algorithms, AIS incorporating the danger theory used pre-determined schemas to define data as either 'safe' or 'dangerous', a mechanism inspired by the ability of the immune system to discriminate between the two types of cell death, apoptosis and necrosis.

The danger theory, first proposed in 1994 [19], describes how the activation of an immune response is controlled by the detection of danger signals emitted from tissue cells killed via unplanned cell death i.e. necrosis. The danger theory states that the detection of nonself proteins, termed antigen, are insufficient to initiate an immune response by helper Tcells. The detection of danger signals acts as a necessary second signal to provide a 'green light' for the production of antibodies by the adaptive immune system. This is based on the concept that without any damage to the host, there is no need to mount an immune response. This is thought to account for phenomena like the presence of so-called 'friendly bacteria' forming the gut flora, and goes some way to account for 'changing self' in states including pregnancy.

The guiding principle of the danger theory is the immune system's ability to sense the difference in cell death by the mechanisms of apoptosis and necrosis. Cells undergo apoptosis for a number of reasons, for controlling the morphology of tissues, to maintain homeostasis, or simply because the cell reaches the end of its lifespan. When a cell undergoes apoptosis it releases chemical messengers into the surrounding fluid, indicating to phagocyte cells that the apoptotic debris requires removal from the tissue. The apoptosing cell shrinks and is absorbed into for example a passing macrophage and all trace of the cell death is absorbed by a process termed phagocytosis. Contrastingly, necrosis is a much more violent process. This is a form of unplanned cell death, occurring due to one of four triggers: extreme heat, extreme cold, lack of oxygen and infection by a pathogen. The cell's membrane rapidly degrades and molecules normally bound behind a cell membrane leak into the interstitial fluid. Examples of such signals include heat shock proteins, uric acid and monosodium urate, products of rapid, uncontrolled decomposition of nuclear materials. These molecules of rapid cell decomposition are termed danger signals, more recently formalised as a group of Danger-Associated Molecular Patterns (or DAMPs) [22].

The cell responsible for processing the signals of necrosis 
and apoptosis is the professional antigen presenting cell, the dendritic cell, which resides in the interstitial fluid of tissues, traversing the area and detecting the presence and absence of signals released as a result of cell death. The release of apoptotic signals initiates the transformation of dendritic cells into a tolerogenic state, instructing the adaptive immune system to behave anergencially towards any current pathogens residing in the tissue. Conversely the detection of the danger signals produced by tissue cell necrosis tansforms the dendritic cell into a potent stimulator of the adaptive immune system, causing helper T-cell activation and subsequent antibody production against any pathogens in the monitored tissue. This differentiation of dendritic cells into either a tolerogenic or activatory state is a key step in controlling immune system responses, controlled by sensing the balance between apoptotic and necrotic signals.

The danger theory is used in a variety of different ways in AIS, but always relies on the encoding of the release of some sort of danger signal, first proposed in [13]. The Dendritic Cell Algorithm [15] is most commonly cited when referring to the use of the danger theory in AIS, although other danger based approaches exist often incorporating a second signal into a self-nonself based system as in [24]. In a tissue growth algorithm [10], a 'pure danger' approach was employed, which does not depend on processing by artificial dendritic cells, nor does it rely on the self-nonself principles. In this paper and that in [5] a pure danger approach is also employed, with the focus firmly on abstract models of the processes of apoptosis and necrosis, without the explicit use of any form of dendritic cell-based agent. As per the previous work on incorporating danger theory into CA's, differentiation between apopotosis and necrosis effects the manner by which the automata grows. Filters are applied to determine if a cell undergoes apoptosis or necrosis and this is then used to determine the evolution of the automata. Our implementation of necrotic filters to the automata controls which type(s) or automata with controlled growth, are permitted to survive. Akin to the detection of multiple types of danger signals, in this study we explore the stacking of necrotic filters in the control of the automata.

\section{DESIGN OF EXPERIMENTS}

\section{A. Evolutionary Algorithm Design}

The evolutionary algorithm in this study, before adding necrosis, is a standard one. The cellular automata updating rules are stored as strings of 36 integers with values in the range $0-7$, specifying cell states. Two variation operators are used: two point crossover of the string and $k$-point mutation that replaces the value at at $k$ positions, selected uniformly at random within the rule. Selection and replacement are accomplished with generational size-four tournament selection. The population is shuffled into groups of four CA-rules. The two more fit are copied over the two less fit. The copies are subjected to crossover and mutation. Such an updating is called a generation. In each experiment, the algorithm is run for 4000 generations with a population of 200 automata rules.
A collection of 30 replicate evolutionary runs with different random number seeds is used in each experiment.

The algorithm is modified as follows to implement necrosis. The entire population is passed, one at a time, to the necrosis test(s) used in that instance of the algorithm. If population members fail this test, they are recognized as emitting danger signals, and their fitness is reduced to zero. Necrosis is applied immediately after reproduction and fitness evaluation of new structures. Both old and new structures are tested for by the necrosis filter. The steps are shown as Algorithm 1, with the one novel step given in blue. The necrosis functions used in the various experiments are given below.

\section{Algorithm 1: Necrotic Filtered EA}

Initialize Population

Main Loop

Evaluate fitness

Test necrotic filter condition(s)

Zero the fitness of failing chromosomes

Perform fitness based selection

Perform reproduction of selected chromosomes

End Loop

\section{B. Necrosis Filters}

Two types of necrosis filters are employed, each in a soft or a crisp form. The first type is shape controlled. Four shapes are employed, covering the drawing arena as shown in Figure 3. The are called the middle half, middle quarter, diamond, and lower gap shapes. The number of live pixels outside of the gray area during the rendering of the automata are counted. Crisp necrosis is applied unconditionally if there are pixels in the white area at all, for soft necrosis the number of living cells $n$ outside of the shape are totalled and a CA rule was tested for emitting danger signals by comparing a uniform random number in the range $[0,1]$ to the function given in Equation 1.

$$
p(n)=\frac{e^{\left(\frac{n}{K}-r\right)}}{e^{\left(\frac{n}{K}-r\right)}+1}
$$

The value $K$ scales the number of cells, $n$, that are out of bounds and $r$ is an offset. The function in Equation 1 is the probability of necrosis, conditioned on the number of cells outside of the shape. The value $K=2000$ are used with $r=2$ The parameter $K$ controls the sharpness of the sigmoid probability curve given by Equation 1 while $r$ is an offset that lowers the chance of necrosis as $r$ increases.

$$
E=-\sum_{i=1}^{m} p_{i} \cdot \log _{2}\left(p_{i}\right)
$$

The second type of necrosis experiment performed was based on Shannon entropy. The number of each type of living state appearing in a rendering are compiled an divided by the total number of living cells to create empirical probabilities $p_{i}$ of use for each living state $i$ appearing in the rendering. The Shannon entropy of this distribution, given in Equation 
Fig. 2. Shown are renderings of time histories of 100 evolved apoptotic cellular automata.

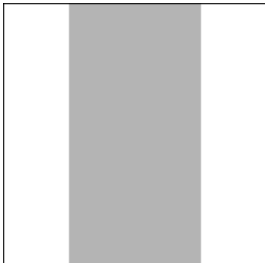

Shape 0

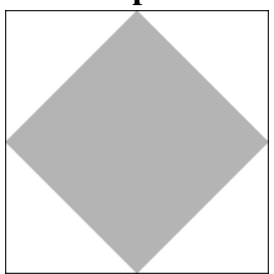

Shape 2

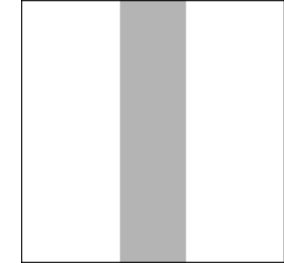

Shape 1

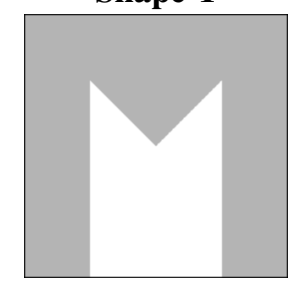

Shape 3
Fig. 3. These are the four shapes used to define necrotic filters, with filters applied to the white and grey areas as detailed in Section III-B

2, is used to perform necrosis. Necrosis is not applied to low fitness (small) rendered automata to prevent necrosis from operating on automata with a small number of living cells. The smallness restriction on applying necrosis was implemented after many runs, with the lowest upper bound on acceptable entropy values, terminated with all individuals of zero fitness. In the crisp version of entropy-based necrosis, an automata rule is found to be emitting danger signals if their entropy exceeds, or fails to exceed, a given bound. In the experiments in this study we set a pre-determined smallness value to avoid necrosis to 200 living cells, i.e. necrosis is not applied to population members with a fitness, that is number of living cells, below 201.

$$
p(n)=\frac{e^{ \pm(\mathcal{E}-\mathcal{S}) / K-Q}}{e^{ \pm(\mathcal{E}-\mathcal{S}) / K-Q}+1}
$$

The soft version of entropic necrosis was also based on the Shannon entropy, using the same sigmoid probability mechanic as the shape based experiment. The sigmoid function used is given in Equation 3, where $\mathcal{E}$ is the entropy bound, $S$ is the observed Shannon entropy of the rendered automata, $K$ controls the sharpness of the sigmoid curve, and $Q$ is an offset. Preliminary experimentation resulted in a choice of $K=1.0$ and $Q=1.0$.

\section{Experiments Performed}

In the earlier study, the experiments that sought to evolve automata with a state entropy of no more than $\mathcal{E}=0.8$. About two-thirds of the runs ended in automata with sizes (fitness) below 201, which permitted them to violate the entropy bound. The entropy necrosis in the earlier study was crisp and so one of the experiments is to re-run crisp entropic necrosis seeking entropy below 0.8 and also run the soft version of entropic necrosis using the same bound.
The earlier study also applied crisp entropic necrosis seeking entropy above 1.0,1.2, and 1.4. There were no failures to reach these entropic lower bounds and the achieved values of entropy suggested that high entropy values are easy to meet. This study applied both crisp and soft necrosis with lower entropy bounds of 1.2, 1.8, and 2.2.

Experiments were run for all four shapes using crisp necrosis with $K=2000$ and $r=2$, soft necrosis with these parameters having been used in the earlier study. Finally an experiment using the middle half shape with $K=2000$ and $r=2$ and entropic necrosis with the goal $E>2.2$ were run using the four possible combinations of hard and soft necrosis; a control run with no necrosis was also performed as a baseline. These experiments test the effect of employing multiple necrosis filters in a single evolutionary run.

\section{RESUlTs AND Discussion}

Figure 4 shows the soft (upper) and crisp (lower) entropic necrosis experiment seeking entropy values below $E=0.8$. Sixteen of the thirty crisp runs failed to meet the entropy bound, echoing results of the earlier study. These failures caused the largest rendered automata have no more than 200 pixels. All of the results using soft necrosis achieved high fitness optima, but only one of them actually met the entropy goal. The actual entropy values, sorted into increasing order, are shown in Figure 5. The purple squares show clearly the runs that did and did not meet the entropic goal. The earlier study demonstrated that entropy values below $E=0.8$ are difficult. This is confirmed by the way that the soft entropic necrosis achieved the bound only once. It is worth noting that the soft entropic necrosis, while failing to meet the upper entropy goal, achieved much lower entropy than the failing runs and much lower entropy than the control runs. Tightening the soft necrosis function's sigmoid curve might allow even lower entropies.

Figure 6 shows the distribution of fitness and entropy values for the experiments seeking entropy above 1.2, 1.8, and 2.2 with both crisp and soft entropic necrosis. The fitness results suggest that all three entropy levels are easy for the evolutionary algorithm, aided by necrosis, to achieve. The entropy plots are more informative. Looking at the entropy values for $E>2.2$, the soft results have a much broader distribution of values. This broadness is not present or less apparent for entropy values 1.2 and 1.8, suggesting that values of 2.2 are starting to become difficult. Of the thirty results for $E=2.2$, eleven did not make it to $E=2.2$. One soft result for $E=1.2$ achieved an entropy of 0.68 , below the desired value - other than that all runs for $E=1.2$ and $E=1.8$ did achieve the bound.

Changing from soft to crisp necrosis when shapes are being used to drive the necrosis was not too different from the soft results, except that the fitness values were slightly (not significantly) lower. Figure 7 shows the four best results 

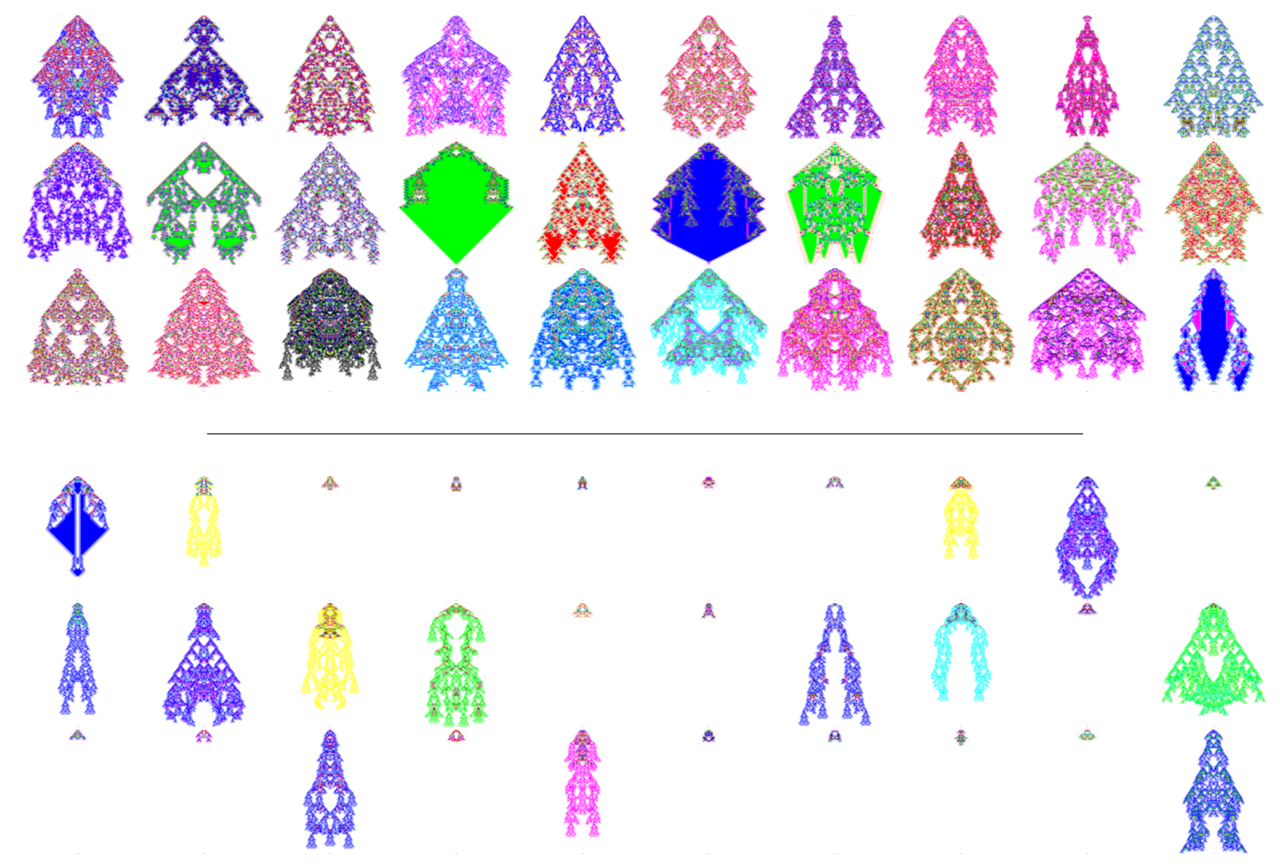

Fig. 4. Shown the 30 evolved apoptotic cellular automata using soft (upper) and crisp (lower) filters for apoptotic CA with state use entropy below 0.8 .

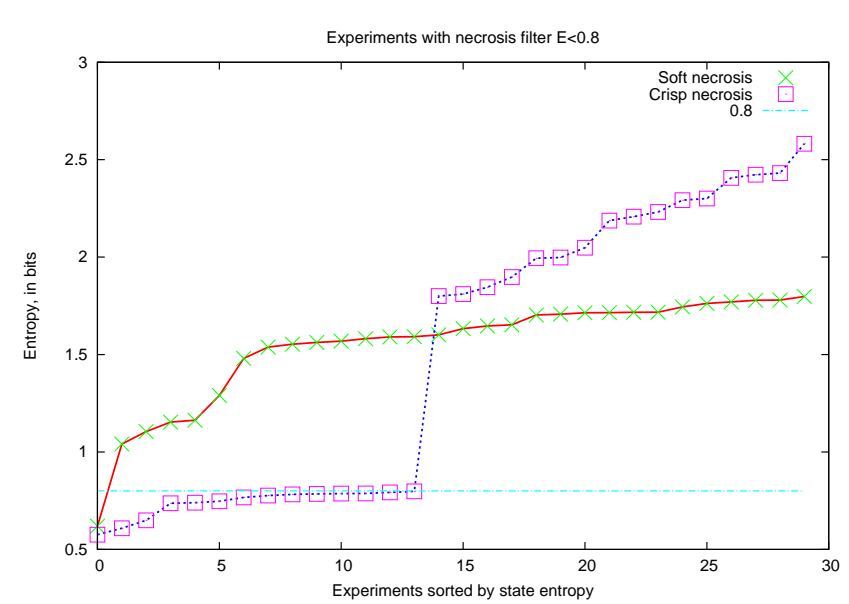

Fig. 5. Shown are the entropy values for all 30 runs using an upper entropy bound of 0.8 with soft and hard necrosis. A horizontal line denotes the position of entropy 0.8 . Crisp runs above this line are the tiny results from Figure 4 that failed to break out.

for the lower-gap shape. These automata renderings do not resemble any appearing in any other run, confirming that the shape based necrosis does exert substantial control in its crisp form, as the earlier study showed similarly in its soft form.

In comparison to the earlier study, the greatest change in the way necrotic filters are applied in this study is in the use of multiple necrotic filters in a single evolutionary run. The simplest shape, the middle half (shape 0), was combined with the somewhat challenging condition $E>2.2$ testing all four combinations of soft and crisp. Entropy of the rendering of an automata does not directly affect fitness, while the middle half shape constraint either places out of bounds or makes less acceptable half of the area that can be used to generate fitness. Figure 8 shows the distribution of fitness and entropy values for a control experiment with no necrotic constraint and the four different stacked shape-entropy experiments.

The most obvious result it that crisp shape constraint lowers fitness significantly: this is exactly what one would expect as the crisp necrosis absolutely 'locks away' half the potential fitness. Making the entropic constraint crisp raised the entropy; the double soft experiment was the only one with entropy more variable than the control experiment. The experiments with soft shape necrosis has fitness similar to the control experiment. These results again indicate the need to experiment more with the shape of the soft necrosis probability curve for entropy-based constraints.

These experiments demonstrate that stacking necrotic filters is practical. While it would be easy to add filters that would choke evolution, the single filter crisp $E<0.8$ half chokes it, it is possible to put filters together without causing a problem. A necrotic filter either excludes (crisp) of de-emphasizes (soft) a portion of the fitness landscape. If filters are stacked, their exclusions of portions of the fitness landscape are intersected. The several earlier papers on evolving apoptotic cellular automata indicate there are a huge number of optima in the search space, leaving lots of room to carve off pieces of it.

Figure 9 shows renderings of the automata from the control experiments and the four stacked necrotic filter experiments. The strongest visual effect is the one that also appears in the fitness distributions, the second and fourth panel of the figure are smaller. The entropy enhancements for the crisp entropic runs are visible, but only to close observation. The fifth panel is the double soft experiment is shown in the last panel. Two renderings have large, solid blue areas, indicating very low entropy. These are the most extreme example of 


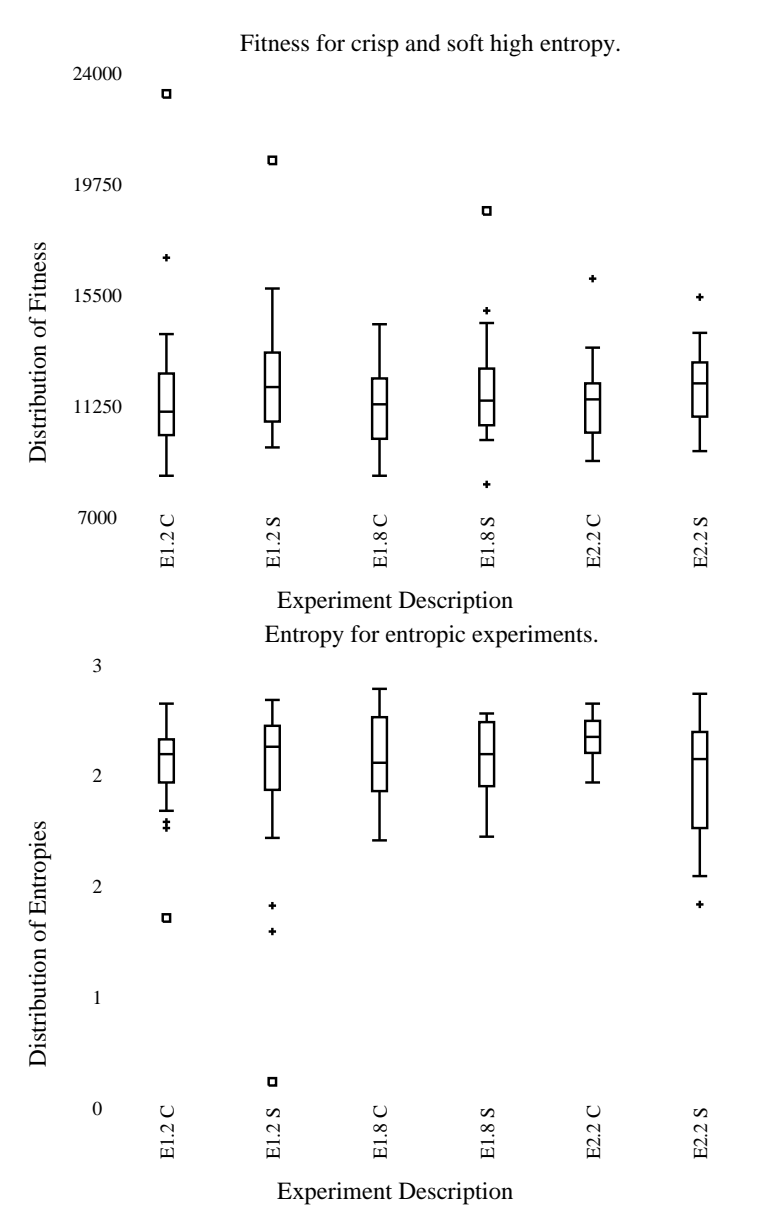

Fig. 6. Shown are the fitness and entropy distributions of the experiment seeking high state entropy using both soft and crisp entropic necrosis.

leakage through the soft filter.

\section{Conclusions And NeXt Steps}

This study demonstrated the practicality of using soft and crisp versions of both the shape and entropic necrotic constraints. It also showed that stacking filters is works well. Experiments established that $E=2.2$ is a high enough lower bound on entropy to be a difficult problem. It is also clear that more experimentation with the control parameters for soft entropic necrotic filters is a good idea. In the limit, the $K$ parameter can transform the soft necrosis into the crisp necrosis. So far only one soft value has been carefully tested.

A significant implication of the research is that we can use necrotic filters to pare away parts of the fitness landscape, effectively excluding undesirable parts of the fitness landscape. While care must be exercised to avoid paring away the entire fitness landscape, necrotic filters can decompose and so enormously simplify the design of fitness functions for evolutionary algorithms. Any detectable flaw can be excluded or discouraged by crisp or soft necrotic constraints. The huge diversity of optima in the space of apoptotic cellular automata makes them a natural test-bed for experimenting with necrotic control of evolution, but it is clear the technique needs to be tested in much broader domains.
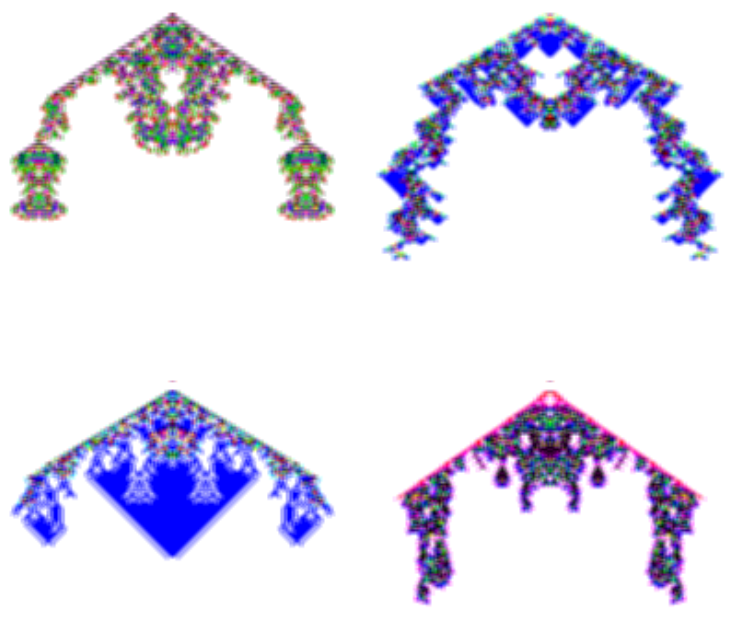

Fig. 7. Example of rendered automata arising from crisp necrosis employing the lower gap shape.

The demonstration that using entropic necrotic filtration to require high state-entropy values established, by the comparison of soft and crisp control results, these techniques could be used to detect the point at which the entropic constraints were becoming hard to satisfy. This suggests that soft-crisp contrasts can be used as stress tests to determine when a constraint excludes a high fraction of the fitness landscape. This also suggests that an interesting question of how large is the intersection of different constraints. This topic could be treated in part by examining other constraints like restriction to a desired band of fitness values or topbottom color contrasts for renderings of apoptotic cellular automata.

An early priority for additional work is to start applying necrotic control to other evolutionary algorithms. It may be possible to have adaptive filters that learn optima of an optimization problem as they are located and act to exclude them in subsequent runs. Another natural target is generative algorithms, where interactions between different commands for constructing solutions often have unexpected effects, making necrotic filtration a natural choice. A broad variety of possible avenues are available for expanding this work.

\section{A. Dynamic Necrotic Control}

Figure 4 highlights the very different impact of soft and crisp necrotic control. This is the current best example of crisp necrotic control strangling evolution to some degree. In contrast, soft necrotic control only produced the desired result one time in thirty. Stacking both these filters would be frivolous - they effect of the crisp filter dominates the effect of the soft one. An alternative to this is to shift between two or more filters over the course of evolution. This would cause the shape of the admissible fitness landscape to change over 

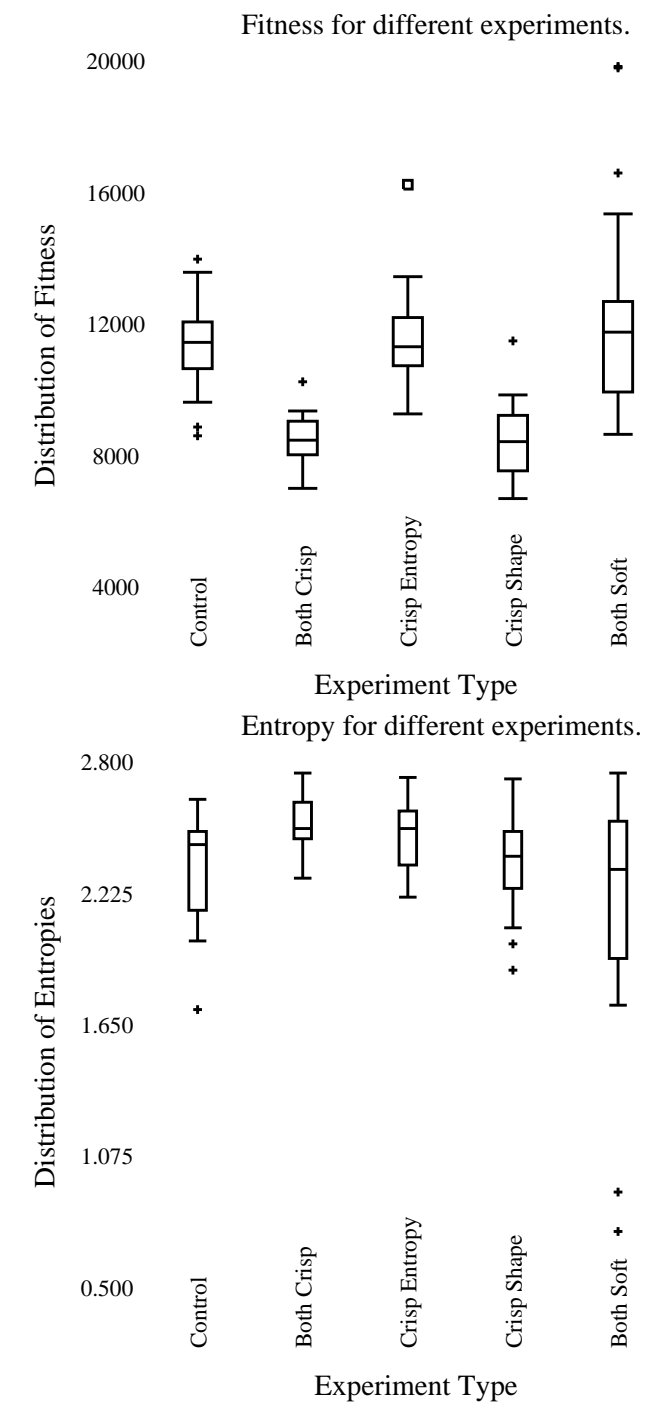

Fig. 8. Shown are the fitness and entropy distributions of the control experiment and experiments with stacked necrotic filters for entropy $e \geq 2.8$ and the middle half shape using both crisp and soft necrosis.

the course of evolution, in effect allowing dynamic necrotic control of evolution.

This notion has the potential to allow better control of evolution, but it also is the standard disaster of evolutionary computation: another tuneable aspect. It may be that soft control followed by crisp control would work well; or the opposite might produce better performance. There is also the question of the time of hand-off from one filter to another. The good value for order, filtration strength, and hand-off times will almost certainly be problem dependent. There are certainly an array of novel avenues to explore with this technique both in terms of understanding the stacking of filters and indeed in the breadth of potential application domains.

\section{REFERENCES}

[1] A. Adamatzky, J. Serquera, and E.R. Miranda. Automata-2008: Theory and Applications of Cellular Automata: "Cellular automata sound synthesis: From histograms to spectrograms". Luniver Press, 2008.

[2] P. Anghelescu. Encryption algorithm using programmable cellular automata. IEEE 2011 World Congress on Internet Security (WorldCIS), pages $233-239,2011$.

[3] D. Ashlock. Evolvable fashion-based cellular automata for generating cavern systems. In Proceedings of the 2015 IEEE Conference on Computatational Intelligence in Games, pages 306-313, 2015.

[4] D. Ashlock and L. Bickley. Rescalable, replayable maps generated with evolved cellular automata. In Acta Physica Polonica (B), Proceedings Supplement, volume 9(1), pages 13-22, 2016.

[5] D. Ashlock and J. Greensmith. Necrotic control of the aesthetics of evolved art. In Proceedings of the 2020 Congress on Evolutionary Computation, pages 1-8, Piscataway NJ, 2020. IEEE Press.

[6] D. Ashlock and B. Jamieson. Evolutionary computation to search mandelbrot sets for aesthetic images. Journal of Mathematics and Art, 1(3):147-158, 2008.

[7] D. Ashlock and S. McNicholas. Fitness landscapes of evolved cellular automata. IEEE Transaction on Evolutionary Computation, 15(2):198212, 2013.

[8] D. Ashlock and C. Pugh. Evolutionary cellular automata bonsai. In Proceedings of the 2013 Congress on Evolutionary Computation, Piscataway, NJ, 2013. IEEE Press.

[9] D. Ashlock and J. Tsang. Evolved art via control of cellular automata. In IEEE Congress on Evolutionary Computation, 2009, pages 3338 3344, May 2009.

[10] P. Bentley, J. Greensmith, and S. Ujjin. Two ways to grow tissue for artificial immune systems. In Proc. of the 4th International Conference on Artificial Immune Systems (ICARIS), LNCS 3627, pages 139-152. Springer-Verlag, 2005.

[11] A.A. Burbelko, E. Fras, W. Kapturkiewicz, and D. Gurgul. Modelling of dendritic growth during unidirectional solidification by the method of cellular automata. Materials Science Forum, 649:217-222, 2010.

[12] A.A. Burbelko and D. Gurgul. Simulation of austenite and graphite growth in ductile iron by means of cellular automata. Archives of Metallurgy and Materials, 55(1):53-60, 2010.

[13] S. Cayzer and U. Aickelin. The danger theory and its application to artificial immune systems. In Proc. of the 1st International Conference on Artificial Immune Systems (ICARIS), pages 141-148. University of Kent at Canterbury Printing Unit, 2002.

[14] J. Garner and D. Ashlock. Evolution of 2D apoptotic cellular automata. In Proceedings of the 2015 Congress on Evolutionary Computation, pages 2160-2167, Piscataway, NJ, 2015. IEEE Press.

[15] J. Greensmith, U. Aickelin, and S. Cayzer. Introducing Dendritic Cells as a novel immune-inspired algorithm for anomaly detection. In Proc. of the 4th International Conference on Artificial Immune Systems (ICARIS), LNCS 3627, pages 153-167. Springer-Verlag, 2005.

[16] J. Greensmith, A. Whitbrook, and U. Aickelin. Artificial Immune Systems. In Handbook of Metaheuristics, pages 421-448. Springer, 2010.

[17] Lawrence Johnson, Georgios N. Yannakakis, and Julian Togelius. Cellular automata for real-time generation of infinite cave levels. In Proceedings of the 2010 Workshop on Procedural Content Generation in Games, PCGames '10, pages 10:1-10:4, New York, NY, USA, 2010. ACM.

[18] M. E. Laragga and L. Alvarez-Icaza. Cellular automaton model for traffic flow based on safe driving policies and human reactions. Physica A, 389(23):5425-5438, 2010.

[19] P. Matzinger. Tolerance, danger and the extended family. Annual Reviews in Immunology, 12:991-1045, 1994.

[20] G. Monro. Emergence and generative art. Leonardo - MIT Press, 42(5):476-477, 2009.

[21] K. Nakamura and K. Imada. Incremental learning of cellular automata for parallel recognition of formal languages. In Proceedings of the 13th international conference on Discovery science, DS'10, pages 117-131, Berlin, Heidelberg, 2010. Springer-Verlag.

[22] T. Pradeu and E.L. Cooper. The danger theory: 20 years later. Frontiers in immunology, 3(287), 2012.

[23] E. Sapin, O. Bailleux, and J. Chabrier. Research of complexity in cellular automata through evolutionary algorithms. Complex Systems, 11, 1997.

[24] S. Sarafijanovic and J. Le Boudec. An artificial immune system for misbehavior detection in mobile ad-hoc networks with virtual thymus, clustering, danger signal and memory detectors. In Proc. of the 3rd International Conference on Artificial Immune Systems (ICARIS), LNCS 3239, pages 342-356, 2004. 

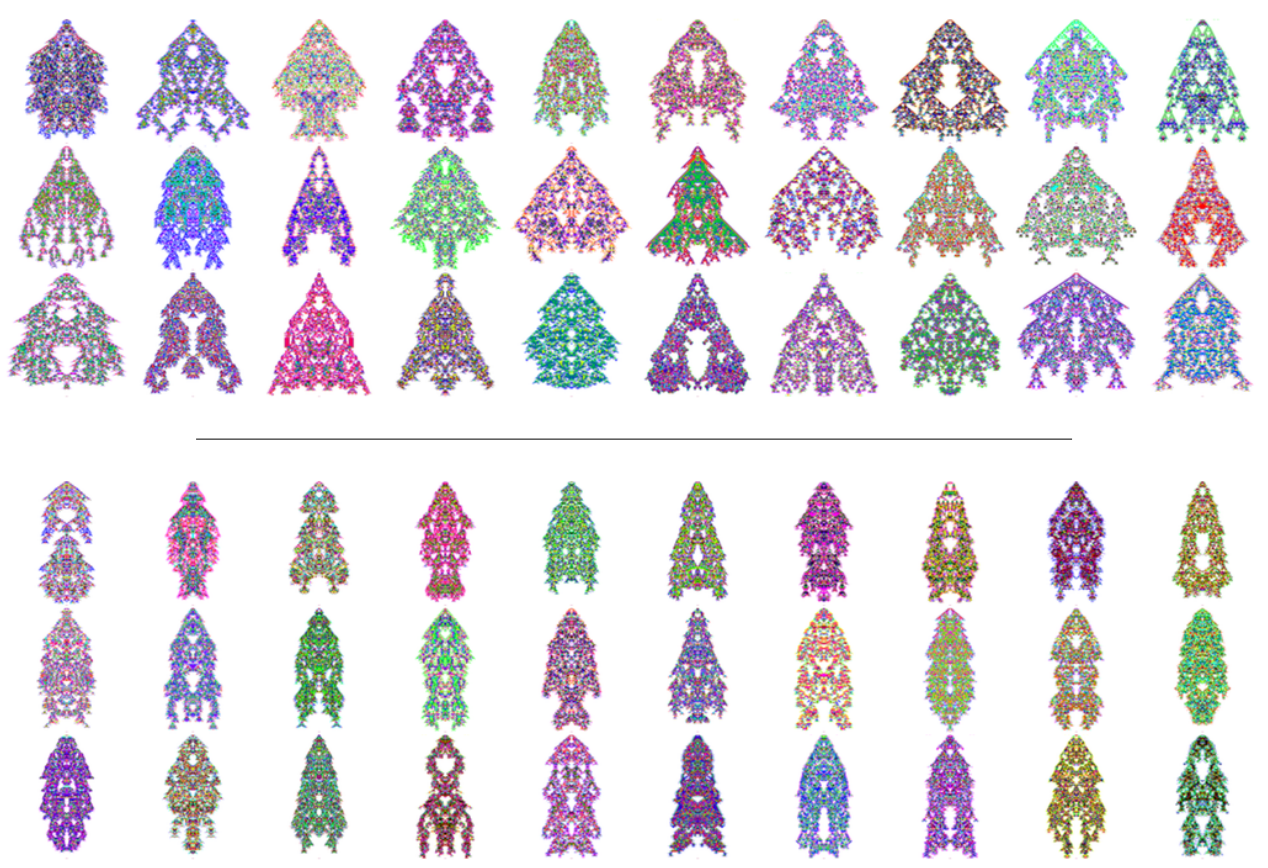

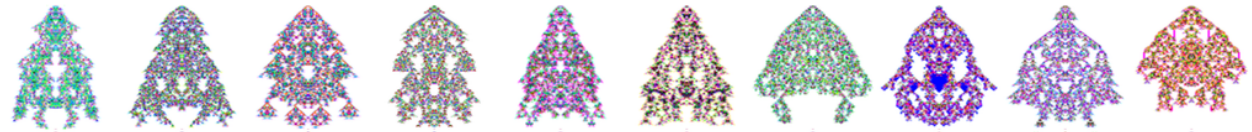
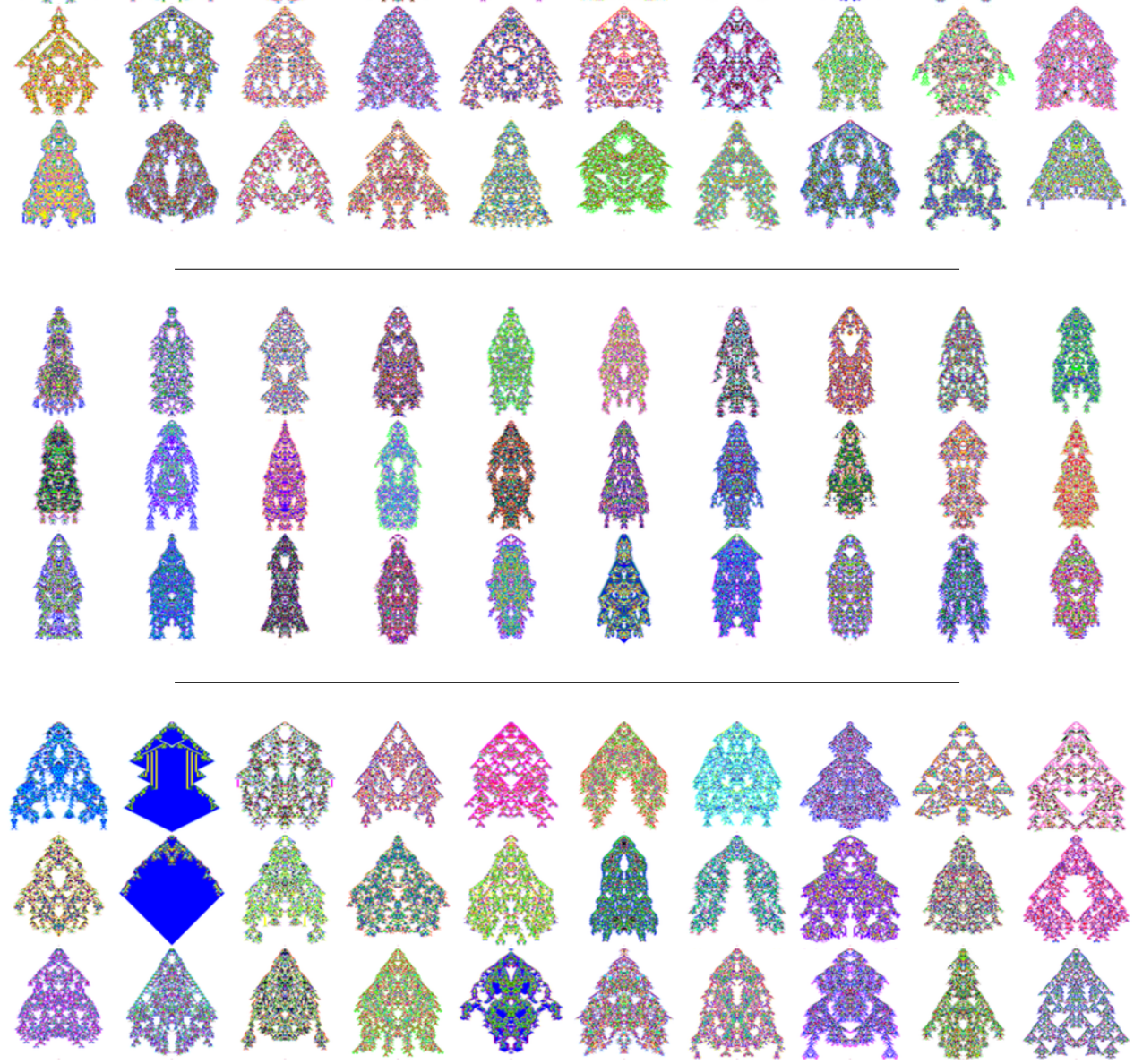

Fig. 9. Shown the 30 evolved apoptotic cellular automata using the necrotic costraints of the middle half shape and entropy of at least 2.2. The top panel has no constraints, the other four use both constrains in the form, from top to bottom, both crisp, entropy crisp, shape crisp, and both constraints soft. 
[25] J. Serquera and E. R. Miranda. Cellular automata sound synthesis with an extended version of the multitype voter model. In Audio Engineering Society Convention 128, 52010.

[26] S. Wolfram. Universality and complexity in cellular automata. Physica D: Nonlinear Phenomena, 10(1-2):1-35, 1984. 